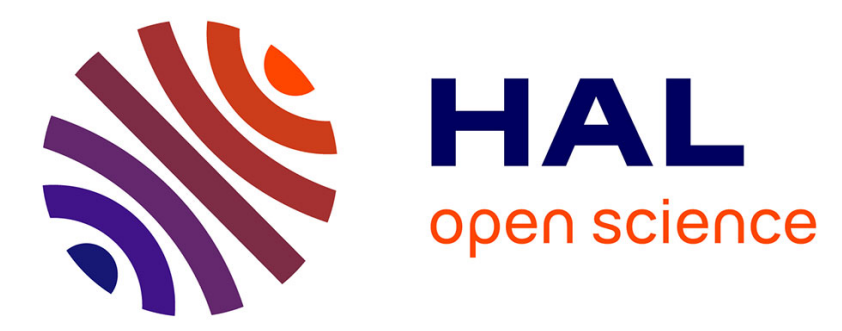

\title{
Historique et cadre des recherches sur le site du Mont Beuvray
}

Jean-Paul Guillaumet, Pierre-Paul Bonenfant, Jean-Loup Flouest, Katherine

Gruel

\section{To cite this version:}

Jean-Paul Guillaumet, Pierre-Paul Bonenfant, Jean-Loup Flouest, Katherine Gruel. Historique et cadre des recherches sur le site du Mont Beuvray. Gallia - Archéologie de la France antique, 1998, L'oppidum de Bibracte, 55, pp.6-8. 10.3406/galia.1998.3256 . hal-01914602

\section{HAL Id: hal-01914602 \\ https://hal.science/hal-01914602}

Submitted on 10 Mar 2020

HAL is a multi-disciplinary open access archive for the deposit and dissemination of scientific research documents, whether they are published or not. The documents may come from teaching and research institutions in France or abroad, or from public or private research centers.
L'archive ouverte pluridisciplinaire HAL, est destinée au dépôt et à la diffusion de documents scientifiques de niveau recherche, publiés ou non, émanant des établissements d'enseignement et de recherche français ou étrangers, des laboratoires publics ou privés.

\section{(ㅇ)(1) $\$$}

Distributed under a Creative Commons Attribution - NonCommercial - NoDerivatives| 4.0 
La synthèse que nous proposons ici insiste plus particulièrement sur les apports de l'analyse stratigraphique et rappelle les points forts des découvertes sur le Mont Beuvray en renvoyant autant que possible aux publications parues ou sous presse.

K. G., J.P. G., P.-P. B.

\section{HISTORIQUE ET CADRE DES RECHERCHES SUR LE SITE DU MONT BEUVRAY}

\section{LES RECHERCHES DE J.-G. BULLIOT ET J. DÉCHELETTE À LA LUMIÈRE DES FOUILLES MODERNES}

L'évolution des problématiques et des techniques de fouilles mises en œuvre entre le milieu du XIX ${ }^{\mathrm{c}}$ s. et la fin du XX ${ }^{\mathrm{e}} \mathrm{s}$. fait du Mont Beuvray un site prédisposé à l'histoire des sciences archéologiques. De fait, l'examen des publications et des inédits que Jacques-Gabriel Bulliot et Joseph Déchelette, les principaux découvreurs de Bibracte, consacrèrent au Mont Beuvray révèle, à l'amont de leurs résultats qui furent parfaitement complémentaires, des objectifs et des méthodes archéologiques nettement différentes.

Jacques-Gabriel Bulliot (1817-1902) consacre ses loisirs à l'étude de l'archéologie et de l'histoire du Morvan. Il se rend acquéreur d'objets qu'il léguera par la suite au musée de la Société éduenne à Autun, dont durant quarante ans, de 1861 à sa mort en 1902, il assure la présidence. Ce sera l'âge d'or de la Société qui achètera l'hôtel Rolin en 1878 afin d'y installer ses collections. En 1851, il présente au Congrès archéologique de France, qui se tient à Nevers, le problème de ce lieu de mémoire collective qu'est le Mont Beurray qui, pour lui, ne saurait être la Bibracte de César, quoi qu'en ait dit une tradition reprise depuis la Renaissance. Tout le monde savant, en effet, s'accorde à reconnaître, depuis la découverte faite à Autun au XVII ${ }^{\mathrm{e}}$ s. d'un ex-voto à la déesse Bibracte ${ }^{2}$, que c'est bien à Autun que se situait l'oppidum de Bibracte, très opulent du temps de César. Ce sont l'étude

2. Sur cette inscription, dont l'authenticité est controversée, voir M. L.ejeune (1990) et C. Rolley (1987). des textes antiques et la recherche épigraphique qui provoquent l'ouverture des fouilles.

Ces fouilles se déroulaient ainsi : Bulliot embauchait des ouvriers et un contremaître dans les villages voisins. Chaque matin il montait sur la Chaume. Les fouilleurs suivaient les murs repérés par des tranchées de sondage ouvertes à la pioche. Sauf dans quelques petits ensembles, le centre de chaque pièce restait intact, afin d'éviter un surcroît de travail. En règle générale, la fouille s'arrêtait au niveau du premier sol rencontré. Parfois le remplissage plus ancien était atteint, en quelques points, sans doute aux endroits où le premier sol n'apparaissait pas clairement. La terre était rejetée sur les bords de la tranchée. Le mobilier découvert était déposé et trié. On récoltait ainsi les monnaies, les objets métalliques, les céramiques décorées et quelques rares pots entiers en céramique grossière. Ce choix reposait principalement sur l'esthétique de chaque pièce.

À partir de cette abondante documentation, Bulliot livre annuellement des comptes rendus dans les Mémoires de la Société éduenne qui sont de véritables rapports de fouilles. Il a en effet une vision très moderne de la documentation de fouilles : "Ensevelies à la fin de chaque campagne sous le remblai, pour rendre le sol aux exploitants, les habitations de Bibracte ne laisseront d'autre souvenir que celui qui est consigné dans les procès-verbaux et les plans de fouilles, c'est pourquoi nous tenons à noter, à cataloguer, maison par maison, tous les détails qui permettent de restituer leur physionomie et leur destination "(Bulliot, 1899, I, p. 116-117). Ces textes sont cependant sommaires quant au travail de synthèse et n'offrent aucune vision globale des types de constructions et de mobiliers. Seuls, quelques articles sur l'émaillerie, l'ex-voto à la Dea Bibracte et les voies du Beuvray développent une approche critique sur ces sujets. Son ouvrage paru en 1899, Les fouilles du mont Beuvray (ancienne Bibracte) de 1867 à 1895, est simplement une réédition de ses comptes rendus annuels repaginés (Bulliot, 1899). Ce n'est en rien une synthèse des travaux comme on a trop souvent tendance à l'imaginer. La vraie nouveauté de cette publication est la création, avec les Thiollier, d'un album de planches photographiques où sont présentés, avec une légende succincte et sans renvoi aux deux volumes de texte, les principaux objets découverts (Thiollier, Thiollier, 1899). Sur ces études celtiques, Bulliot, avec l'aide de J. Roidot, s'essaya à une seule synthèse. Un premier tome uniquement, intitulé La Cité 
gauloise (Bulliot, Roidot), parut en 1879, bien qu'élaboré en 1865-1869. C'est une monographie sur le monde celtique inspirée du célèbre ouvrage de Fustel de Coulanges La Cité antique. Il n'en a hélas ni le souffle ni la qualité. Ce premier tome devait être suivi d'un second appelé $L a$ Cité éduenne, pour lequel nous avons pu retrouver le plan et quelques projets d'illustration. La lecture de ce plan montre que ce travail se base sur les textes, les données historiques et légendaires et très peu sur les découvertes archéologiques. On peut y voir une des raisons de l'abandon du projet de publication. Lorsque paraît en 1879 le premier tome, le second, à la lumière des recherches de terrain en cours sur le Beuvray, n'est plus d'actualité. Aussi, Bulliot extrait les parties informatives prévues pour les chapitres $4,5,16,17$, et les publie dans les Mémoires de la Société éduenne et dans les volumes rééditant ses écrits parus en 1899. Avant tout, Bulliot se considéra toujours comme le découvreur de Bibracte et c'est ainsi également que son neveu Déchelette le verra.

Joseph Déchelette est davantage un homme de cabinet. Sa première publication traitant du Mont Beuvray est l'Inventaire général des monnaies antiques recueillies au Mont Beuvray de 1867 à 1898 (Déchelette, 1899). C'est un travail de recherche sur les provenances et les datations, sans aucun souci de lcur répartition sur lc sitc. Scs deux publications suivantes seront capitales et montreront une ampleur de vue exceptionnelle, fondée sur une méthode comparative extrêmement efficace : il s'agit de deux courtes synthèses sur les habitats et le mobilier de Bibracte; elles ont pour but de montrer les similitudes des découvertes avec celles des autres oppida de l'Europe moyenne jusqu'à l'Europe centrale. C'est la découverte de la "civilisation des oppida», emblématique de la fin de l'époque laténienne. En 1903, J. Déchelette écrit le premier guide consacré au Beuvray (Déchelette, 1903) : on peut le considérer comme l'unique synthèse couvrant l'ensemble des travaux de fouilles sur la montagne depuis 1867. La publication de ses propres recherches sera faite en 1904 (Déchelette, 1904). Ce n'est pas une simple mise en ordre de ses notes de fouilles, mais une synthèse élaborée de ses recherches. Il y traite autant des structures que du mobilier et conclut par des questions de chronologie.

Bulliot, régionaliste convaincu, passionné par l'histoire de sa ville, Autun, et de ses origines, retrouve Bibracte presque seul et, au début, contre tous. Ses fouilles se sont prolongées des années durant. Il a laissé une documentation considérable mais aucune synthèse ni étude d'ensemble. Déchelette se place, en revanche, dès sa première étude concernant Bibracte, sur un autre registre. Il publie des catalogues, des résumés de données et réalise relativement moins de fouilles. C'est par la perspicacité de ses comparaisons typologiques qu'il fait de Bibracte un site de référence européen. Il couronne ainsi le travail de son oncle qui « poursuivit, avec un dévouement aussi constant que modeste, ses travaux sur le sommet du Beuvray et mérita la reconnaissance des Éduens et des savants. » Bulliot, dès qu'il s'essaie à la synthèse, raisonne suivant les méthodes historiques de l'époque, tandis que Déchelette invente une méthode de critique des matériaux archéologiques afin de parvenir, avec leur aide, à écrire l'histoire.

Certes les techniques de fouilles ont bien changé (y compris d'ailleurs entre les premières et les dernières fouilles de Bulliot) et les subtilités de la stratigraphie ont largement échappé aux terrassiers de ces deux savants, mais la qualité des plans, levés en partie par des topographes de l'armée, est indéniable. Cependant, si les plans sont cohérents secteur par secteur, on ne peut se fier à leur orientation, calée sur le Nord magnétique, qui reste approximative : ceci explique la difficulté rencontrée aujourd'hui encore pour déterminer les grands axes de circulation et établir les liens entre les quartiers. Par ailleurs, Bulliot et Déchelette furent surtout attentifs à l'organisation spatiale du site, explorant par tranchées de vastes étendues mais négligeant le plus souvent l'approche stratigraphique, de sorte que le plan de synthèse de leurs travaux (fig. 2) reflète seulement l'état le plus récent des constructions dans chaque secteur. De même, Déchelette ne parvint pas à cerner précisément la durée de l'occupation de l'oppidum - qu'il limite à la seconde moitié du I ${ }^{\mathrm{rr}} \mathrm{s}$. avant J.-C. - et, surtout, il s'abstient de classer le mobilier en différentes séquences, ce qui a eu pendant longtemps des conséquences néfastes sur l'établissement de la chronologie de la fin de la période laténienne et du début de l'époque romaine. Très heureusement, les anciens fouilleurs ont souvent arrêté leur investigation au premier sol construit, préservant ainsi les couches archéologiques plus profondes. C'est ainsi que nous ne connaissions avant la reprise des fouilles à la Porte du Rebout qu'un état, celui du murus gallicus final, alors que cinq fortifications successives sont aujourd'hui discernées, que la domus PC 1 du Parc aux Chevaux ne se limite plus à une seule construction mais à plusieurs édi- 
fices successifs, que les aménagements de la Fontaine Saint-Pierre se sont succédé de la fin de l'époque gauloise au $\mathrm{XIX}^{\mathrm{e}} \mathrm{s} .$. L'étude conjointe des stratigraphies et des « ensembles clos " de mobilier livrés par les fouilles récentes permet enfin de subdiviser l'occupation antique du site en plusieurs horizons chronologiques qui s'échelonnent de la seconde moitié du II's. avant J.-C. au règne d'Auguste.

J.-P. G., P.-P. B.

(Jean-Paul Guillaumet, Pierre-Paul Bonenfant)

\section{LA REPRISE DES FOUILLES DEPUIS 1984}

Depuis 1984, une douzaine d'équipes dirigées par des universitaires et des chercheurs a été amenée à fouiller au Mont Beuvray. Ces équipes interviennent généralement un mois chacune, ce qui représente environ de 3500 à 4500 journées ouvrées par an. Ces travaux de terrain sont intégrés dans le cursus des universités européennes participantes. De 1988 à 1993, le programme européen Erasmus, porté d'abord par l'université Paris-I (O. Buchsenschutz) puis par l'École du Louvre (A. Duval), a concrétisé cette coopération interuniversitaire pour la formation pratique des étudiants en archéologie.

Une équipe du CNRS (l'URA 33, aujourd'hui UMR 126) effectue en 1984 un premier sondage dans le rempart, à la Porte du Rebout, pour évaluer la complexité des fortifications. En 1985, un deuxième programme de fouilles est lancé sur les sanctuaires de la Chaume. À partir de 1986, l'opération est ouverte à d'autres équipes, tant françaises qu'étrangères.

Tout en achevant les programmes déjà lancés précédemment, il est décidé dès 1987 de concentrer les nouvelles équipes sur la fouille d'une zone d'habitat le long de l'axe montant de la Porte du Kebout à la Pâture du Couvent et au Parc aux Chevaux. L'équipe du CNRS précitée engage ainsi une fouille étendue sur la Pâture du Couvent, tandis que l'université de Lausanne (Suisse) entame en 1988 un sondage stratigraphique sur la domus PC 1. L'arrivée d'H. Richard, du laboratoire de Chronoécologie de Besançon (actuelle UMR 9946 du CNRS), entraîne l'ouverture d'un chantier en milieu humide, à la Fontaine Saint-Pierre, lié aux problématiques environnementales. Par ailleurs, les fouilles de la Chaume se poursuivent par l'exploration de l'espace contigu de la Terrasse. Les fouilles de la Pâture du Couvent, encore inachevées, ont pris une grande ampleur et deviennent le principal chantier, avec le dégagement d'une des voies principales de l'oppidum, ornée d'un bassin monumental, de deux îlots de constructions du I ${ }^{\mathrm{er}} \mathrm{s}$. avant J.-C., ainsi que des ruines d'un couvent franciscain.

De 1992 à 1994, les différents travaux nécessaires à la construction du musée de Bibracte ont aussi entrâné de multiples surveillances et des fouilles de sauvetage assurées par l'équipe permanente du Centre archéologique du Mont Beuvray, notamment la mise au jour d'une des nécropoles de l'oppidum ( $c f$. infra, p. 43). Une petite équipe d'archéologues salariés a par ailleurs été chargée, en 1994 et 1995, de préciser la localisation de constructions fouillées au XIX' $\mathrm{x}$. sur le secteur central de l'oppidum. Quant aux prospections géophysiques, elles ont porté au total sur 8 ha et les relevés topographiques divers sur 11 ha environ. Parmi ces derniers, il faut mentionner tout particulièrement le positionnement par F. Schubert du rempart extérieur, découvert en 1985, sur un fond de plan photogrammétrique au 1/2500.

Parallèlement aux recherches de terrain, des chercheurs ont pris en charge l'étude globale de certains types de mobiliers archéologiques ou de documents. Une première partie de ces travaux a été consacrée à la documentation ancienne de Bulliot et Déchelette (notamment Laubenheimer, 1991 ; Guillaumet, 1996, qui font suite à Guillaumet, 1979 et 1984). Dans une deuxième étape, ces recherches ont pris en compte les données des fouilles récentes, qui ne sont devenues suffisantes pour justifier les premières synthèses qu'à partir du début des années 1990.

Onze ans après la reprise des recherches, les découvertes archéologiques ont considérablement modifié les caractères du site tels qu'ils avaient pu être définis par Bulliot et Déchelette.

J.-L. F., K. G. (Jean-Loup Flouest, Katherine Gruel)

\section{L'OCCUPATION DU SITE ET SON CONTEXTE ENVIRONNEMENTAL}

\section{L'ENVIRONNEMENT}

Dès le début du programme engagé sur l'oppidum de Bibracte, il a été clairement établi que les thèmes de recherches principaux devaient être replacés dans leurs 